\title{
Corrections to the Standard Model of Cosmology
}

\author{
Joel Smoller*, Blake Temple*, And Zeke Vogler* \\ Dedicated to our friend Marshall Slemrod
}

\begin{abstract}
In this note, which is a first step in authors' longer calculation, we present a system of equations for computing the $p=0$ evolution of corrections to the Standard Model of Cosmology consistent with corrections produced by spherical self-similar perturbations of the Standard Model known to exist during the radiation phase of the Big Bang. We record here that the asymptotic equations close at leading order, creating a subsystem which contains a stable rest point which does not correspond to the unperturbed critical zero pressure Friedmann spacetime. Corrections to the Standard Model lying within the domain of attraction of this stable rest point would produce, time-asymptotically, quadratic corrections to redshift vs luminosity, i.e., quadratic corrections to the Hubble constant going out from the center, that would be independent of initial conditions, and different from the predictions of the Standard Model. In future investigations authors will characterize the parameter values associated with self-similar waves from the radiation era that would give rise to corrections that lie within this domain of attraction. The results in this paper arose as a step in the authors' program to compute the corrections to redshift vs luminosity produced by self-similar waves from the radiation epoch, interpreting this as a baseline mechanism for the creation of anomalous accelerations that does not require extraneous fields like Dark Energy. Details will appear in their forthcoming paper.
\end{abstract}

\section{Introduction}

We present a system of equations for computing the evolution of corrections to the Standard Model of Cosmology which are consistent with corrections produced by spherical self-similar waves known to exist during the radiation phase of the Big Bang. Following ideas set out by Glimm and Lax

*Temple and Vogler supported in part by NSF Applied Mathematics Grant Number DMS-040-6096. Smoller supported in part by NSF Applied Mathematics Grant Number DMS-189-1105. 
in the theory of conservation laws, our starting point of view is the idea that during the radiation phase, when the pressure satisfies the equation of state $p=\rho c^{2} / 3$, (lasting up until the pressure drops to zero around $10^{5}$ years after the Big Bang), the evolution equations of general relativity form a highly nonlinear system of conservation laws for which we can expect local fluctuations to decay to simple non-interacting wave patterns. That is, the equation of state closes off the field of contact discontinuities, and the remaining characteristic fields have a large sound speed and modulus of genuine nonlinearity, implying that all characteristic fields contribute strongly to decay in the sense of Glimm and Lax, [8, 9, 12]. Time asymptotic wave patterns would consist of multiple "elementary waves", and our view is that the one parameter family of spherical self-similar waves known to perturb the critical, $k=0, p=\rho c^{2} / 3$ Friedmann spacetime, are the most plausible candidates for elementary waves that would be created in a neighborhood of the center. Like the Standard Model, such a correction to the Standard Model would create a Hubble constant consistent with critical expansion at zero order near the center, but would introduce quadratic and cubic corrections going out from the center. Because it is based on the rigid constraints of the Einstein equations, requiring no extraneous fields like the Cosmological Constant or Dark Energy, this wave theory represents the simplest possible physical mechanism for the creation of an anomalous acceleration. Thus, whether or not this mechanism is sufficient to explain in full the anomalous acceleration of the galaxies, it represents a baseline theory to which all other theories of the anomalous acceleration can be compared. The authors are currently constructing a framework in which to compute the corrections to redshift vs luminosity predicted at present time by this wave model, and our calculation requires asymptotic equations for the corrections after the pressure drops to zero.

In this paper we present a clean formulation of the $p=0$ evolution equations, and then use these exact equations to derive a closed system of asymptotic equations which apply to a special class of corrections to the critically expanding $p=0$ Standard Model sufficiently general to include initial data arising from corrections associated with a one parameter family of $p=c^{2} / 3 \rho$ self-similar waves that perturb the critical Friedmann spacetime. In a bit of a surprise to the authors, we report here that the asymptotic equations, which close at the leading order, contain a stable rest point which does not correspond to the unperturbed, $k=0, p=0$, Friedmann spacetime. The presence of the rest point implies that spherical corrections to the Standard Model that lie within the domain of attraction of this stable 
rest point, would produce, time-asymptotically, quadratic corrections to redshift vs luminosity, i.e., corrections to the Hubble constant near the center, that would be independent of the initial perturbations, and different from the predictions of the Standard Model. Now when the pressure drops to zero, one would expect the Standard Model to be sensitive to corrections determined from an earlier epoch when the pressure was large. From this perspective, it is surprising to discover a stable rest point different from the Standard Model, as this implies evolution toward a universal quadratic correction that is insensitive to earlier perturbations. Keep in mind, the stability of the rest point is not to general perturbations of the equations, but is a more subtle leading order stability to perturbations consistent with selfsimilar waves from an earlier epoch when mechanisms are in place to create such waves. In a forthcoming paper, authors will explore parameter values of self-similar waves from the radiation epoch, (determined by the so-called acceleration parameter $a,[18]$ ), that would produce corrections within the domain of attraction of this rest point. The work here arose as part of the authors' program to compute the corrections to redshift vs luminosity at present time produced by self-similar waves from the radiation epoch of the Big Bang [17], and the results supply motivation and clarification for these investigations. Details will appear in authors' forthcoming paper.

\section{Background}

In the Standard Model of Cosmology, on the largest scale, (about a tenth of the distance across the visible universe), after the radiation epoch, (some $10^{5}$ years after the Big Bang), it is considered a reasonable assumption that the pressure drops to approximately zero, and the universe is modeled by a critically expanding, ( $k=0$, implying zero spatial curvature), zero pressure $p=0$ Friedmann-Robertson-Walker (FRW) spacetime,

$$
d s^{2}=-d t^{2}+R(t)^{2}\left\{d r^{2}+r^{2} d \Omega^{2}\right\},
$$

where $d \Omega^{2}$ denotes the line element on the 2 -sphere, $[13,19]$. Here $R(t)$ is the cosmological scale factor, and according to (1),

$$
\bar{r} \equiv R(t) r
$$

measures radial arclength distance at each fixed time. 
In the case when $p=\sigma \rho, 0 \leq \sigma \leq 1 / 3, R(t)$ increases in forward time according to

$$
R(t)=\left(\frac{t}{t_{0}}\right)^{\frac{2}{3(1+\sigma)}}
$$

the density $\rho(t)$ decreases according to

$$
\rho(t)=\frac{4}{3 \kappa(1+\sigma)^{2}} \frac{1}{t^{2}},
$$

the Hubble constant $H(t)=\frac{\dot{R}(t)}{R(t)}$ decreases according to

$$
\frac{H(t)}{H_{0}(t)}=\frac{t_{0}}{t},
$$

the age of the universe is

$$
t=\frac{2}{3(1+\sigma)} \frac{1}{H(t)}
$$

and the horizon distance, (the "size" of the visible universe) at fixed $t$ is given by

$$
r_{\infty}=\frac{2}{1+3 \sigma} \frac{1}{H(t)}
$$

$[15,19]$. Here time $t$ is measured by time since the Big Bang such that $R(0)=0$ and $R\left(t_{0}\right)=1$, and this depends on $\sigma$ through (6). In the Standard Model at present time, the expanding universe of galaxies provides the fluid, assumed to be of uniform density $\rho$ at each fixed time, following co-moving paths $d r / d t=0$, an assumption that is taken as a model of the expanding universe of galaxies valid on length scales about a tenth of the distance across the visible universe. The formulas (1)-(7) follow from the assumption that (1) solves the Einstein equations

$$
G=\kappa T
$$

assuming a perfect fluid stess energy tensor $T$ of the form

$$
T^{i j}=(\rho+p) u^{i} u^{j}+p g^{i j},
$$


with $p=\sigma \rho$. The resulting equations (8) for the FRW spacetime then take the form

$$
\begin{gathered}
H^{2}=\frac{\kappa}{3} \rho, \\
\dot{\rho}=-3(\rho+p) H,
\end{gathered}
$$

which close in $\rho$ and $H$ once the equation of state $p=p(\rho)$ is specified. Equations (1)-(7) follow from (10) upon assuming $p=\sigma \rho$. Although the coordinates identify a center of the spacetime at $r=0$, the 3 -space at each fixed time is zero curvature $k=0$ Euclidean space, implying that the FRW metric (1) is invariant under translation of the center, $[19,15]$. Corrections to the $p=\sigma=0$ FRW Standard Model (1)-(7) are required to account for the quadratic corrections to red-shift vs luminosity observed in the supernova data, $[5,6,7,11]$. This is most often accounted for by some fluid-like notion of Dark Energy, modeled by a constant negative pressure-like term $\Lambda g^{i j}$ added to the right hand side of (9), where $\Lambda$ is the Cosmological Constant, $[19,18]$. Dark Energy is otherwise unobserved, and must account for some seventy percent of the energy density of the universe to produce an effect consistent with the observed anomalous acceleration, [1, 2]. Other measured effects of general relativity have so far not required such a term. Our main goal is to investigate the most plausible mechanism for the anomalous acceleration that does not require the addition of any extra terms to Einstein's original equations.

In this note we present equations for the evolution of spherical corrections to the critical, $k=0, p=0$, FRW Standard Model based on a selfsimilar variable $\xi=r / t$ that provides a measure of fractional distance to the horizon at each fixed time. Expanding about the center in this variable, we derive asymptotic equations for the corrections described by a simple system of four ODE's, and show that the asymptotics close at both the second and fourth orders in the expansion. Our starting point is the following theorem. (Details will appear in our forthcoming paper.)

Theorem 1. Assume the equation of state $p=\sigma \rho$, and define the change of coordinates

$$
\bar{t}=F(\eta) t ; \quad \bar{r}=\eta t,
$$

where

$$
\eta=\frac{\bar{r}}{t},
$$


and

$$
F \equiv F(\eta)=\left(1-\frac{\alpha(\alpha-2)}{4} \eta^{2}\right)^{-\frac{1}{\alpha-2}}
$$

Then in $(\bar{t}, \bar{r})$ coordinates, the $p=\sigma \rho$ solution (1)-(7) takes the form

$$
d s^{2}=-\frac{\left(1-\frac{\alpha(\alpha-2)}{4} \eta^{2}\right)^{2 \frac{\alpha-1}{\alpha-2}}}{\left(1-\left(\frac{\alpha \eta}{2}\right)^{2}\right)} d \vec{t}^{2}+\frac{d \bar{r}^{2}}{\left(1-\left(\frac{\alpha \eta}{2}\right)^{2}\right)}+\bar{r}^{2} d \Omega^{2}
$$

where $\alpha$ is the convenient parameter

$$
\alpha=\frac{4}{3(1+\sigma)},
$$

so that by (3),

$$
R(t)=\left(\frac{t}{t_{0}}\right)^{\alpha / 2}
$$

Theorem 1 gives a representation of the $p=\sigma \rho$ FRW spacetimes in Standard Schwarzschild Coordinates (SSC) where the metric takes the general form

$$
d s^{2}=-B(\bar{t}, \bar{r}) d \bar{t}^{2}+\frac{1}{A(\bar{t}, \bar{r})} d \bar{r}^{2}+\bar{r}^{2} d \Omega^{2}
$$

but in the case of (15) the metric components $A$ and $B$ are especially nice because they are self-similar in the sense they depend only on the self-similar variable $\eta=\bar{r} / t$. Note that by (12)

$$
\xi=\frac{\bar{r}}{\bar{t}}=\frac{\eta}{F(\eta)}
$$

so (15) is self-similar in the variable $\xi$ as well. In the case $\sigma \neq 0$, it is known that these spacetimes admit self-similar perturbations, first identified in the case $p=\rho c^{2} / 3$ in the profoundly original paper of Cahill and Taub [3]. Following this, an exhaustive classification of self-similar solutions of the Einstein equations was set out by Carr and Coley, c.f. [4] and references therein. The authors rediscovered these solutions independently in a search for local time asymptotic waves in [18]. Of interest to us is the case $p=$ 
$\sigma=0$, the only value of $\sigma$ which does not admit a family of self-similar perturbations of (15), c.f. [4] and Theorem 6 below.

In the case $p=\sigma=0, \alpha=4 / 3, F(\eta)=\left(1+\frac{2}{9} \eta^{2}\right)^{3 / 2}$, and the metric (15) reduces to

$$
d s^{2}=-\frac{\left(1+\frac{2}{9} \eta^{2}\right)^{3 / 2}}{\left(1-\left(\frac{2 \eta}{3}\right)^{2}\right)} d \vec{t}^{2}+\frac{d \bar{r}^{2}}{\left(1-\left(\frac{2 \eta}{3}\right)^{2}\right)}+\bar{r}^{2} d \Omega^{2} .
$$

Letting $A_{F}, B_{F}, \rho_{F}$ and $v_{F}$ denote the FRW values in this case, we obtain the $p=0$ Standard Model formulas

$$
\begin{gathered}
A_{F}=1-\left(\frac{2 \eta}{3}\right)^{2}, \\
B_{F}=\frac{1}{\left(1+\frac{2}{9} \eta^{2}\right)\left(1-\left(\frac{2 \eta}{3}\right)^{2}\right)}, \\
\kappa \rho_{F} \bar{r}^{2}=\frac{4}{3} \eta^{2}, \\
v_{F}=\frac{\bar{u}^{1}}{\bar{u}^{0}} \frac{1}{\sqrt{A_{F} B_{F}}} .
\end{gathered}
$$

where for (23) we have used (13) in (4).

Now since $\bar{r}$ is arclength distance at fixed time and $t$ is time since the Big Bang, it follows from (6) and (7) that $\eta=\bar{r} c t$, and hence $\xi$, are natural dimensionless variables to expand in because they are a measure of the fractional distance to the Hubble length $c / H$, the distance traveled by light starting at the Big Bang. That is, $c / H$ is a measure of the distance to the horizon, which is a measure of the size of the visible universe. We now expand $A_{F}, B_{F}, \rho_{F}$ and $v_{F}$ in powers of $\xi$, and then look for equations describing corrections to the Standard Model in powers of $\xi$. This will lead to a satisfactory set of equations for the corrections valid near the center of the spacetime, where our self-similar waves should apply.

Since $A_{F}, B_{F}$ and $\rho_{F} \bar{r}^{2}$ are given in terms of $\eta$, we get $\eta$ in terms of $\xi$ by inverting

$$
\xi=\frac{\eta}{F(\eta)}=\frac{\eta}{\left(1+\frac{2}{9} \eta^{2}\right)^{3 / 2}} .
$$


For this write the exact expression

$$
\eta^{2}=\xi^{2}\left(1+\frac{2}{9} \eta^{2}\right)^{3}=\xi^{2}+\frac{2}{3} \eta^{2} \xi^{2}+\frac{2}{3^{3}} \eta^{4} \xi^{2}+\frac{2^{3}}{3^{6}} \eta^{6} \xi^{2} .
$$

Since we know $\eta^{2}=\xi^{2}+\frac{2}{3} \xi^{4}+O\left(\xi^{6}\right)$, we get

$$
\eta^{2}=\xi^{2}+\frac{2}{3} \xi^{4}+\frac{14}{27} \xi^{6}+O\left(\xi^{8}\right) .
$$

Putting this into (25) and continuing this way we can get the expansion to all orders. Thus we get

$$
B_{F}=\frac{A_{F}=1-\frac{4}{9} \xi^{2}-\frac{8}{27} \xi^{4}+O\left(\xi^{6}\right),}{\left(1+\frac{2}{9} \xi^{2}\right)\left(1-\left(\frac{2 \xi}{3}\right)^{2}\right)+O\left(\xi^{4}\right)},
$$

or

$$
B_{F}=1+\frac{2}{9} \xi^{2}+O\left(\xi^{4}\right)
$$

and,

$$
\rho_{F} \bar{r}^{2}=\frac{4}{3 \kappa} \xi^{2}+O\left(\xi^{4}\right) .
$$

It remains to expand $v_{F}$ in powers of $\xi$. Using

$$
\begin{gathered}
v_{F}=\frac{\bar{u}^{1}}{\bar{u}^{0}} \frac{1}{\sqrt{A_{F} B_{F}}}, \\
B_{F}=\frac{1}{\left(1+\frac{2}{9} \xi^{2}\right) A_{F}}+O\left(\xi^{4}\right),
\end{gathered}
$$

we have

$$
A_{F} B_{F}=\frac{1}{1+\frac{2}{9} \xi^{2}}+O\left(\xi^{4}\right)
$$

and so

$$
\sqrt{A_{F} B_{F}}=1-\frac{1}{9} \xi^{2}+O\left(\xi^{4}\right) .
$$


Now

$$
\begin{aligned}
\frac{\partial \bar{x}^{\alpha}}{\partial x^{i}}=J^{-1} & =\left(\begin{array}{cc}
\left(\frac{\alpha}{2}-1\right) \eta F^{\prime}(\eta)+F(\eta) & t^{\alpha / 2} F^{\prime}(\eta) \\
\frac{\alpha}{2} \eta & t^{\alpha / 2}
\end{array}\right) \\
& =\left(\begin{array}{cc}
-\frac{1}{3} \eta F^{\prime}(\eta)+F(\eta) & t^{2 / 3} F^{\prime}(\eta) \\
\frac{2}{3} \eta & t^{2 / 3}
\end{array}\right) .
\end{aligned}
$$

Thus

$$
\begin{aligned}
\bar{u}^{\alpha}=\frac{\partial \bar{x}^{\alpha}}{\partial x^{i}} u^{i} & =\left(\begin{array}{cc}
-\frac{1}{3} \eta F^{\prime}(\eta)+F(\eta) & t^{2 / 3} F^{\prime}(\eta) \\
\frac{2}{3} \eta & t^{2 / 3}
\end{array}\right)\left(\begin{array}{l}
1 \\
0
\end{array}\right) \\
& =\left(\begin{array}{c}
-\frac{1}{3} \eta F^{\prime}(\eta)+F(\eta) \\
\frac{2}{3} \eta
\end{array}\right) .
\end{aligned}
$$

Using

$$
\begin{array}{r}
\eta=\xi+\frac{1}{3} \xi^{3}+O\left(\xi^{5}\right), \\
\xi=\eta-\frac{1}{3} \eta^{3}+O\left(\xi^{5}\right), \\
F(\eta)=1+\frac{1}{3} \xi^{2}+O\left(\xi^{4}\right),
\end{array}
$$

we obtain

$$
\begin{aligned}
F^{\prime}(\eta)=\frac{d F}{d \xi} \frac{d \xi}{d \eta} & =\left(\frac{2}{3} \xi+O\left(\xi^{3}\right)\right)\left(1-\eta^{2}+O\left(\xi^{3}\right)\right) \\
& =\left(\frac{2}{3} \xi+O\left(\xi^{3}\right)\right)\left(1-\xi^{2}\right)+O\left(\xi^{3}\right) \\
& =\frac{2}{3} \xi+O\left(\xi^{3}\right)
\end{aligned}
$$

and

$$
\begin{aligned}
\bar{u}^{0}=-\frac{1}{3} \eta F^{\prime}(\eta)+F(\eta)= & -\frac{1}{3}\left(\xi+\frac{1}{3} \xi^{3}+O\left(\xi^{4}\right)\right)\left(\frac{2}{3} \xi+O\left(\xi^{3}\right)\right) \\
& +1+\frac{1}{3} \xi^{2}+O\left(\xi^{4}\right) \\
= & 1+\left(\frac{1}{3}-\frac{2}{9}\right) \xi^{2}+O\left(\xi^{4}\right) \\
= & 1+\frac{1}{9} \xi^{2}+O\left(\xi^{4}\right)
\end{aligned}
$$


and

$$
\bar{u}^{1}=\frac{2}{3} \eta=\frac{2}{3} \xi\left(1+\frac{1}{3} \xi^{2}\right)+O\left(\xi^{5}\right) .
$$

Putting these into (31) gives

$$
\overline{\mathbf{u}}=\left(\begin{array}{c}
\bar{u}^{0} \\
\bar{u}^{1}
\end{array}\right)=\left(\begin{array}{c}
1+\frac{1}{9} \xi^{2} \\
\frac{2}{3} \xi+\frac{2}{9} \xi^{3}
\end{array}\right)+O\left(\xi^{5}\right) .
$$

Thus we obtain the expansion of $v_{F}$ in terms of $\xi$,

$$
\begin{aligned}
\bar{v}_{F} & =\frac{\bar{u}^{1}}{\bar{u}^{0}} \frac{1}{\sqrt{A B}}=\frac{2}{3} \frac{\xi\left(1+\frac{1}{3} \xi^{2}\right)}{\left(1+\frac{1}{9} \xi^{2}\right)\left(1-\frac{1}{9} \xi^{2}\right)}+O\left(\xi^{4}\right) \\
& =\frac{2}{3} \xi\left(1+\frac{1}{3} \xi^{2}\right)+O\left(\xi^{5}\right) .
\end{aligned}
$$

In the next section we derive equations for corrections to $A_{F}, B_{F}, \rho_{F}$ and $v_{F}$.

\section{The $p=0$ equations}

Since we will now only consider SSC spacetime coordinate $(\bar{t}, \bar{r})$ and will no longer consider FRW comoving coordinates $(t, r)$, we simplify notation by removing the bars from $\bar{t}$ and $\bar{r}$ and will let $t$ and $r$ denote SSC coordinates $\bar{t}$ and $\bar{r}$, respectively. With this notation, assume that the variables $A, B, \rho, v$ of a time dependent SSC metric (18) are given as functions of $(t, \xi), \xi=$ $r / t \equiv \bar{r} / \bar{t}$, and define the new variables

$$
\begin{gathered}
D=\sqrt{A B} \\
z=\frac{c^{2} \rho r^{2}}{1-\frac{v^{2}}{c^{2}}}, \\
w=v / \xi .
\end{gathered}
$$

Then the following theorem gives a formulation of the Einstein equations (8) for the evolution of time dependent SSC spacetime metrics of form (18) in self-similar coordinates $(\bar{t}, \xi)$, assuming $p=\sigma=0$ :

Theorem 2. Assume $p=0$ and the SSC metric form (18). Then the following system of equations is equivalent to the Einstein equations (8):

$$
t z_{t}+\xi\{(-1+D w) z\}_{\xi}=-D w z
$$




$$
\begin{gathered}
t w_{t}+\xi(-1+D w) w_{\xi} \\
=w-D\left\{w^{2}+\frac{1-A}{2 A \xi^{2}}\left(1-\xi^{2} w^{2}\right)\right\}, \\
\xi A_{\xi}=(1-A)-z \\
\xi D_{\xi}=\frac{D}{2 A}\left\{2(1-A)-z+\xi^{2} w^{2} z\right\}
\end{gathered}
$$

Proof: Equations (36)-(39) follow from the locally inertial formulation of the SSC Einstein equations derived in [10], upon setting $p=0$ and making the appropriate substitutions. Details will appear in the authors' forthcoming paper.

Using the formulas (27),(28), (29), (30), and (32), we obtain the following expansions for the $p=0$ Standard FRW metric in terms of the variables $z, w, A, D$ :

Theorem 3. The Standard Model $A=A_{F}(\xi), D \equiv D_{F}(\xi)=\sqrt{A_{F} B_{F}}$, $z \equiv z_{F}(\xi), w \equiv w_{F}(\xi)=v_{F}(\xi) / \xi$ is an exact, time independent solution of equations (36)-(39). These are given in in powers of $\xi$ by the expressions:

$$
\begin{aligned}
z_{F} & =\frac{4}{3} \xi^{2}+\frac{40}{27} \xi^{4}+O\left(\xi^{6}\right) \\
w_{F} & =\frac{2}{3}+\frac{1}{3} \xi^{2}+O\left(\xi^{4}\right) \\
A_{F} & =1-\frac{4}{9} \xi^{2}-\frac{8}{27} \xi^{4}+O\left(\xi^{6}\right) \\
D_{F} & =1-\frac{1}{9} \xi^{2}+O\left(\xi^{4}\right) .
\end{aligned}
$$

Proof: That $A_{F}, D_{F}, z_{F}, w_{F}$ solve equations (36)-(39) is inherent in the equivalence of (36)-(39) with the Einstein equations, or by direct substitution. (Details are left to the reader.) Equations (40)-(43) follow directly from $(27),(28),(29),(30)$, and (32), except we must get $z_{F}$ to fourth order. For this use equation

$$
\xi A_{\xi}^{F}=\left(1-A_{F}\right)-z_{F},
$$

with

$$
A_{F}=1-\frac{4}{9} \xi^{2}-\frac{8}{27} \xi^{4}
$$

and

$$
z_{F}=\frac{4}{3} \xi^{2}+z_{4}^{F} \xi^{4},
$$


in $\xi A_{\xi}^{F}=\left(1-A_{F}\right)-z_{F}$ to solve for $z_{4}^{F}$, and then check consistency with the $z$-equation (36). To fourth order in $\xi$ equation $\xi A_{\xi}^{F}=\left(1-A_{F}\right)-z_{F}$ reads

$$
\xi\left(1-\frac{4}{9} \xi^{2}-\frac{8}{27} \xi^{4}\right)_{\xi}=\frac{4}{9} \xi^{2}+\frac{8}{27} \xi^{4}-\frac{4}{3} \xi^{2}-z_{4}^{F} \xi^{4},
$$

which reduces to

$$
-\frac{8}{9} \xi^{2}-\frac{32}{27} \xi^{4}=-\frac{8}{9} \xi^{2}+\left(\frac{8}{27}-z_{2}^{F}\right) \xi^{4}
$$

leading to

$$
z_{4}^{F}=\frac{40}{27}
$$

Conclude: $z_{F}(\xi)$ to fourth order is given by

$$
z_{F}(\xi)=\frac{4}{3} \xi^{2}+\frac{40}{27} \xi^{4}+O\left(\xi^{6}\right)
$$

Finally, note that since $p=0$ is the equation for dust, there is no interaction of fluid particles except through gravitation. On the other hand, the SSC metric form is invariant under arbitrary transformations of the time coordinate, i.e., choice of guage. The following theorem confirms that the evolution of solutions of (36), (37) reduces to an ODE at the center $\xi=0$, determined by the choice of guage and values of $A$ and $D$ determined by (38) and (39). As a consequence, by proper choice of gauge and initial conditions, solutions can be set to agree with the evolution of the $p=0$ FRW spacetime at the center. We record this as a theorem:

Theorem 4. General solutions of (36)-(39) reduce to an ODE at $r=$ $0=\xi$.

Proof: Since $\xi$ sits in front of the $\xi$-derivative in equations (36)-(39), one can set $\xi=0$ consistently and the resulting evolution equations (36), (37) reduce to an ODE at center.

From this we conclude that the evolution of the "center" of the wave is determined by the initial condition at the center. This is consistent with the fact that everything in the wave is going outward, and there is no interaction with the center when $p=0$. 


\section{Perturbations of the Standard Model}

In this section we derive equations for the evolution of corrections near the center of the $p=0$ Standard Model $A_{F}, D_{F}, z_{F}, w_{F}$ (given in (40)-(43)), consistent with the Einstein equations (36)-(39). That is, we determine equations for the corrections to (40)-(43) in each power of $\xi$. The expansions of $A_{F}, D_{F}, z_{F}, w_{F}$, as well as the ansatz for the corrections $\hat{A}, \hat{D}, \hat{z}, \hat{w}$ given in the equations (45)-(48) below, only involve even powers of $\xi$. This is sufficient to evolve corrections starting from initial data at the end of the radiation phase, induced by self-similar waves that perturb FRW during the radiation phase when $p=\rho c^{2} / 3,[18]$. Our goal now is to prove that the equations close within this ansatz. For our ansatz we take

$$
\begin{aligned}
A(t, \xi)=A_{F}(\xi)+\hat{A}(t, \xi), & \hat{A}=A_{2}(t) \xi^{2}+A_{4}(t) \xi^{4}, \\
D(t, \xi)=D_{F}(\xi)+\hat{D}(t, \xi), & \hat{D}=D_{2}(t) \xi^{2}, \\
z(t, \xi)=z_{F}(\xi)+\hat{z}(t, \xi), & \hat{z}=z_{2}(t) \xi^{2}+z_{4}(t) \xi^{4}, \\
w(t, \xi)=w_{F}(\xi)+\hat{w}(t, \xi), & \hat{w}=w_{0}(t)+w_{2}(t) \xi^{2},
\end{aligned}
$$

where all higher order terms are neglected. Our notation is that subscript or superscript $F$ indicates $p=0 \mathrm{FRW}$, and we use subscript unless other subscripts are present, in which case we raise $F$ to a superscript. Our ansatz is chosen so that only even powers of $\xi$ appear, in agreement with FRW and with [18]. The ultimate justification for this ansatz is that the asymptotic equations derived below close within the terms of this ansatz.

That the ansatz closes requires proof, the details of which will be given in our forthcoming paper. However, to see that it must close, note that the $\xi^{2}$ and $\xi^{4}$ order terms from equation (38), both linear, give $A_{2} \equiv A_{2}(t)$ and $A_{4} \equiv A_{4}(t)$ (c.f. (45)) in terms of $z_{2} \equiv z_{2}(t)$ and $z_{4} \equiv z_{4}(t)$ (c.f. (47)). That is they give

$$
\begin{aligned}
& f_{A 2}\left(A_{2}, z_{2}\right)=0, \\
& f_{A 4}\left(A_{4}, z_{4}\right)=0,
\end{aligned}
$$

for some functions $f_{A 2}, f_{A 4}$ to be determined, from which we can solve for $A_{2}$ in terms of $z_{2}$ and $A_{4}$ in terms of $z_{4}$. Next we can take equation (39) at second order to solve for $D_{2} \equiv D_{2}(t)$ in terms of $z_{2}$, (c.f. (46)), by a relation of the form

$$
f_{D 2}\left(z_{2}, D_{2}\right)=0
$$

because $w$ and $z$ don't enter this equation until the fourth order level. But because of the term $\frac{1-A}{2 A \xi^{2}}$ in the $w$ equation, $A_{2}$ enters the $w$ equation at 
the zero order level, so there is a nontrivial $w_{0}$ equation. For this reason, we must get equations for $w_{0}$ and $w_{2}$, but everything closes at that level. In particular, we will see that $z_{2}$ is constant except for dependence on $w_{0}$. Next the $z$-equation at second and fourth order will give dynamical equations for $\dot{z}_{2}$ and $\dot{z}_{4}$, of form

$$
\begin{gathered}
t \dot{z}_{2}=f_{z 2}\left(z_{2}, z_{4}, w_{0}, w_{2}\right), \\
t \dot{z}_{4}=f_{z 4}\left(z_{2}, z_{4}, w_{0}, w_{2}\right) .
\end{gathered}
$$

Finally the $w$-equation will close the system with two equations

$$
\begin{gathered}
t \dot{w}_{0}=f_{w 0}\left(z_{2}, z_{4}, w_{0}, w_{2}\right), \\
t \dot{w}_{2}=f_{w 2}\left(z_{2}, z_{4}, w_{0}, w_{2}\right),
\end{gathered}
$$

(with $w_{i}=w_{i}(t)$, c.f. (48)), completing the system to a $4 \times 4$ ODE in $\left(z_{2}, z_{4}, w_{0}, w_{2}\right)$. It remains to determine the functions $f_{\alpha}$. This is given in the following theorem, the main result of this section, (details will appear in our forthcoming paper):

Theorem 5. The Einstein equations (36)-(39) imply that the corrections $z_{2}, z_{4}, w_{0}, w_{2}, A_{2}, A_{4}, D_{2}$ evolve according to the following system of ODEs that close in $z_{2}, z_{4}, w_{0}, w_{2}$ :

$$
\begin{aligned}
-t \dot{z}_{2}= & 3 w_{0}\left(\frac{4}{3}+z_{2}\right) \\
-t \dot{z}_{4}=5\left\{\frac{2}{27} z_{2}-\frac{1}{18} z_{2}^{2}\right. & \left.+\frac{4}{3} w_{2}+z_{2} w_{2}\right\} \\
+ & +5 w_{0}\left\{\frac{36}{27}-\frac{2}{9} z_{2}-\frac{1}{12} z_{2}^{2}+z_{4}\right\}
\end{aligned}
$$

where for the last equation

$$
\begin{aligned}
\{\cdot\}_{w 21}=\left\{( \frac { 2 } { 3 } + w _ { 0 } ) ^ { 2 } \left(-\frac{1}{9}\right.\right. & \left.+D_{2}\right)+\left(\frac{2}{3}+w_{0}\right)\left(\frac{2}{9}+w_{2}\right) \\
& \left.+\left(-\frac{1}{3}+w_{0}\right)\left(\frac{2}{3}+3 w_{2}\right)\right\}
\end{aligned}
$$


(56) $\{\cdot\}_{w 22}=\frac{1}{2}\left\{\left(\frac{4}{9}-A_{2}\right)\left(-\frac{1}{9}+D_{2}\right)+\left(\frac{4}{9}-A_{2}\right)^{2}+\left(\frac{8}{27}-A_{4}\right)\right.$

$$
\left.-\left(\frac{4}{9}-A_{2}\right)\left(\frac{2}{3}+w_{0}\right)^{2}\right\} \text {. }
$$

Moreover, the following three constraint equations are determined by the ansatz, and required to close the equations, will hold for all time, assuming they are satisfied on the initial data:

$$
\begin{aligned}
A_{2} & =-\frac{1}{3} z_{2}, \\
A_{4} & =-\frac{1}{5} z_{4}, \\
D_{2} & =-\frac{1}{12} z_{2} .
\end{aligned}
$$

Equations (57)-(59) are the Einstein constraint equations expressed in the coordinate gauge of our ansatz. That is, equations (51)-(54) are equations for corrections to the Standard Model expressed in the SSC coordinates in which the $p=0 F R W$ metric is self-similar, and this requires a particular choice of time coordinate, or gauge, thereby eliminating the guage freedom of SSC. The point is that since the corrections $z_{2}, z_{4}, w_{0}, w_{2}, A_{2}, A_{4}, D_{2}$ are corrections to FRW in a particular coordinate system, unlike the general SSC metric and Einstein equations (36)-(39), the equations (57)-(59) for the corrections are no longer invariant under arbitrary changes of time coordinates. Fixing the gauge essentially fixes the constraints (57)-(59). As a consequence, in order to use equations (57)-(59) to evolve corrections to the Standard Model from initial data taken at the end of the radiation epoch, it is necessary to post-process the initial data by a time transformation (gauge transformation) sufficient to meet the constraints (57)-(59). Although not an issue in this paper, this is a technical problem that must be addressed in our forthcoming paper.

Proof: Equations (51)-(59) follow upon substituting the ansatz (45)-(48) into equations (36)-(39). Details will appear in authors' forthcoming paper.

Note that because the $k=0, p=0$, FRW solution solves equations (36)(39) exactly, it follows that the right hand sides of (51)-(54) must vanish when $z_{i}=w_{i}=0, i=1,2$, and this is a consistency check easily confirmed. Substituting (57)-(59) into (51)-(54), the right hand side of (51)-(54) closes 
up in the variables $U=\left(z_{2}, z_{4}, w_{0}, w_{2}\right)$. If we further make the substitution $\tau=\ln t$, system (51)-(54) takes the homogeneous autonomous form

$$
-t \frac{D U}{d t}=-\frac{d U}{d \tau}=F(U),
$$

where $F(0)=0$. This is quite nice for numerical simulations as an evolution from the end of radiation to present time requires that $t$ range from about

$$
10^{4} \leq t \leq 10^{10},
$$

but in the variable $\tau$,

$$
4.6 \approx 4 \ln 10 \leq \tau \leq 10 \ln 10 \approx 23,
$$

thereby reducing the longtime simulation problem to a triviality. The resulting system of autonomous ODE's is the topic of the next section.

\section{Analysis of the asymptotic equations}

Substituting (57)-(59) into (51)-(54) and simplifying yields the following system of four ODE's in the variables $U=\left(z_{2}, z_{4}, w_{0}, w_{2}\right)$ :

Our system then takes the final form:

$$
\begin{aligned}
&-t \dot{z}_{2}=3 w_{0}\left(\frac{4}{3}+z_{2}\right) \\
&-t \dot{z}_{4}=-5\left\{\frac{2}{27} z_{2}+\frac{4}{3} w_{2}-\frac{1}{18} z_{2}^{2}+z_{2} w_{2}\right\} \\
& \quad-5 w_{0}\left\{\frac{4}{3}-\frac{2}{9} z_{2}+z_{4}-\frac{1}{12} z_{2}^{2}\right\} \\
&-t \dot{w}_{0}=\frac{1}{6} z_{2}+\frac{1}{3} w_{0}+w_{0}^{2}, \\
&-t \dot{w}_{2}=\frac{1}{10} z_{4}+\frac{4}{9} w_{0}-\frac{1}{3} w_{2}+\frac{1}{24} z_{2}^{2}-\frac{1}{3} z_{2} w_{0} \\
& \quad-\frac{1}{3} w_{0}^{2}+4 w_{0} w_{2}-\frac{1}{4} w_{0}^{2} z_{2} .
\end{aligned}
$$

Upon solving (60)-(63) the metric components are determined to appropriate order by (57)-(59)

We find it interesting that our asymptotics close within an ansatz that includes initial data consistent with the self-similar waves that extend the 
Standard Model when $p=\frac{1}{3} \rho$, but yet the ansatz does not admit selfsimilar waves other than the FRW metric when $p=0$. Indeed, for time independent perturbations, (60) implies that $w_{0}=0,\left(z_{2}=-4 / 3\right.$ is not a small perturbation of the Standard Model), so (62) implies that $z_{2}=0$, and using these in (61),(63) implies $z_{4}=w_{2}=0$ as well. This is the main step in proving the following theorem, c.f. [4]

Theorem 6. The only self-similar SSC solution of the Einstein equations that perturbs the Standard Model within the ansatz (45)-(48) when $p=0$, is the $p=0$ FRW spacetime (15).

Again, this contrasts with the case $\sigma \neq 0$ which does admit self-similar perturbations of FRW, (c.f. [18] for the case $p=\frac{1}{3} \rho$ and c.f. [4] for the case of general $\sigma$.)

We can check the stability of the Standard Model within this ansatz by checking the eigenvalues of $D F(U)$ at $U=\left(z_{2}, z_{4}, w_{0}, w_{4}\right)=0$. The linear part of the RHS of (60)-(63) is

$$
t\left(\begin{array}{c}
z_{2} \\
z_{4} \\
w_{0} \\
w_{2}
\end{array}\right)_{t}=\left(\begin{array}{cccc}
0 & 0 & -4 & 0 \\
10 / 27 & 0 & 20 / 3 & 20 / 3 \\
-1 / 6 & 0 & -1 / 3 & 0 \\
0 & -1 / 10 & -4 / 9 & 1 / 3
\end{array}\right)\left(\begin{array}{c}
z_{2} \\
z_{4} \\
w_{0} \\
w_{2}
\end{array}\right) .
$$

To check stability, we calculate the determinant

$$
\begin{aligned}
\operatorname{Det}\left(\begin{array}{cccc}
0 & 0 & -4 & 0 \\
10 / 27 & 0 & 20 / 3 & 20 / 3 \\
-1 / 6 & 0 & -1 / 3 & 0 \\
0 & -1 / 10 & -4 / 9 & 1 / 3
\end{array}\right) \\
=(4)\left|\begin{array}{ccc}
-10 / 27 & 0 & -20 / 3 \\
1 / 6 & 0 & 0 \\
0 & 1 / 10 & -1 / 3
\end{array}\right| \\
=(4)\left(-\frac{1}{6}\right)\left|\begin{array}{cc}
0 & -20 / 3 \\
1 / 10 & -1 / 3
\end{array}\right| \\
=-\left(\frac{2}{3}\right)\left(\frac{20}{3}\right)\left(\frac{1}{10}\right)=-4 / 9<0 .
\end{aligned}
$$

We conclude that the product of the four eigenvalues is negative, so the Standard Model $\left(z_{2}, z_{4}, w_{0}, w_{4}\right)=0$ is unstable. This is not surprising as 
one would expect the $p=0$ corrections at present time would be affected by corrections determined from an earlier epoch when the pressure was nonzero.

A MAPLE calculation gives

$$
\operatorname{Det}\left(\begin{array}{cccc}
-\lambda & 0 & -4 & 0 \\
10 / 27 & -\lambda & 20 / 3 & 20 / 3 \\
-1 / 6 & 0 & -1 / 3-\lambda & 0 \\
0 & -1 / 10 & -4 / 9 & 1 / 3-\lambda
\end{array}\right)=\lambda^{4}-\frac{1}{9} \lambda^{2}+\frac{4}{9} \lambda-\frac{4}{9}
$$

so the roots of the characteristic polynomial $p_{\lambda}=\lambda^{4}-\frac{1}{9} \lambda^{2}+\frac{4}{9} \lambda-\frac{4}{9}$ give the four eigenvalues. Another MAPLE calculation gives the eigenvalue and eigenvector pairs as:

$$
\begin{aligned}
& \lambda_{1}=\frac{2}{3}, \quad R_{1}=\left(\begin{array}{c}
\frac{36}{5} \\
2 \\
-\frac{6}{5} \\
1
\end{array}\right) \\
& \lambda_{2}=-1, \quad R_{2}=\left(\begin{array}{c}
-\frac{108}{5} \\
\frac{112}{3} \\
-\frac{27}{5} \\
1
\end{array}\right) \\
& \lambda_{3}=\frac{1}{6}-\frac{\sqrt{23}}{6} i, \quad R_{3}=\left(\begin{array}{c}
0 \\
1 \\
0 \\
\frac{1}{40}+\frac{\sqrt{23}}{40} i
\end{array}\right) \text {; } \\
& \lambda_{4}=\frac{1}{6}+\frac{\sqrt{23}}{6} i, \quad R_{4}=\left(\begin{array}{c}
0 \\
1 \\
0 \\
\frac{1}{40}-\frac{\sqrt{23}}{40} i
\end{array}\right) \text {. }
\end{aligned}
$$

Now the ODE's (60)-(63) also close up at leading order in the $\left(z_{2}, w_{0}\right)$ variables to form the $2 \times 2$ autonomous system

$$
\begin{aligned}
t \dot{z}_{2} & =-3 w_{0}\left(\frac{4}{3}+z_{2}\right)=f\left(z_{2}, w_{0}\right) \\
t \dot{w}_{0} & =-\frac{1}{6} z_{2}-\frac{1}{3} w_{0}-w_{0}^{2}=g\left(z_{2}, w_{0}\right),
\end{aligned}
$$


whose phase portrait can be solved exactly. That is,

$$
f\left(z_{2}, w_{0}\right)=-3 w_{0}\left(\frac{4}{3}+z_{2}\right)=0
$$

gives the $z_{2}$-isocline as

$$
f\left(z_{2}, w_{0}\right)=0 \text { iff } w_{0}=0 \text { or } z_{0}=-\frac{4}{3}
$$

and

$$
g\left(z_{2}, w_{0}\right)=-\frac{1}{6} z_{2}-\frac{1}{3} w_{0}-w_{0}^{2}=0
$$

gives the $w_{0}$-isocline as

$$
g\left(z_{2}, w_{0}\right)=0 \text { iff } z_{2}=-2 w_{0}\left(1+3 w_{0}\right)
$$

Thus the isoclines intersect in the three rest points of the system,

$$
(0,0),\left(-\frac{4}{3}, \frac{1}{3}\right),\left(-\frac{4}{3},-\frac{2}{3}\right) \text {. }
$$

Now by $(64)$ and $(67)$ we know $(0,0)$ is a saddle with stable direction

$$
\overrightarrow{\left(z_{0}, w_{0}\right)}=\overrightarrow{\left(\frac{108}{5}, \frac{27}{5}\right)}=\overrightarrow{(4,1)}
$$

and unstable direction

$$
\overrightarrow{\left(z_{0}, w_{0}\right)}=\overrightarrow{\left(\frac{36}{5},-\frac{6}{5}\right)}=\overrightarrow{(6,-1)},
$$

and it is not difficult to check that rest point

$$
\left(z_{2}, w_{0}\right)=\left(-\frac{4}{3}, \frac{1}{3}\right)
$$

is a stable node, and

$$
\left(z_{2}, w_{0}\right)=\left(-\frac{4}{3},-\frac{2}{3}\right)
$$

is an unstable node, c.f. Figure 1. 


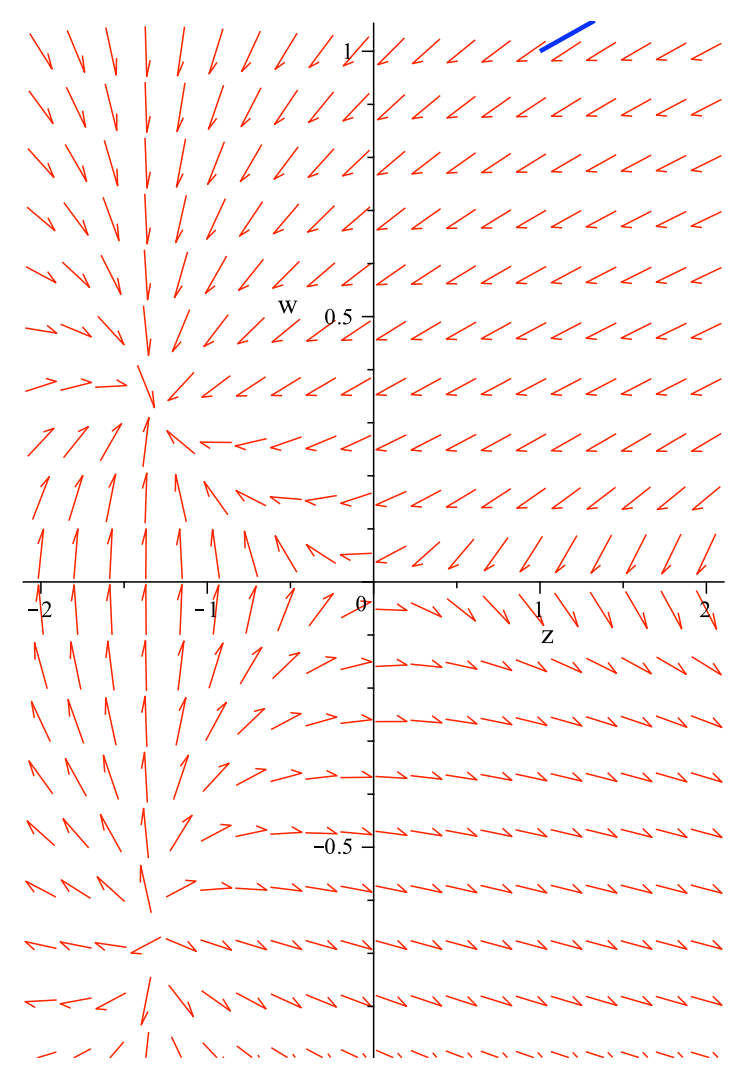

Figure 1: The $\left(z_{2}, w_{0}\right)$ Phase Portrait.

Note, in particular, that the phase portrait shows that any trajectory that perturbes $(0,0)$ above its stable manifold, will tend toward the stable rest point

$$
\left(z_{2}, w_{0}\right)=\left(-\frac{4}{3}, \frac{1}{3}\right) .
$$

It turns out that at the lowest order in $\xi$ correction to the Standard Model, the corrections within the domain of attraction of this rest point determine quadratic corrections to redshift vs luminosity that are (time-asymptotically) independent of time. Said differently, such perturbations of the self-similar $p=0$ Standard Model would tend to a nearby stable self-similar solution, at the leading order near the center $\xi=0$.

Assuming the leading order corrections $z_{2}$ and $w_{0}$ are exactly at the stable rest point, we can put these values into the $z_{4}$ and $w_{2}$ equations to 
get the phase portrait of these higher order corrections at the rest point. Thus, putting

$$
\left(z_{2}, w_{0}\right)=(-4 / 3,1 / 3)
$$

into equations (61) and (63) and simplifying yields the linear autonomous ODE

$$
\left(\begin{array}{c}
\dot{z}_{4} \\
\dot{w}_{2}
\end{array}\right)=\left(\begin{array}{rr}
\frac{5}{3} & 0 \\
-\frac{1}{10} & -1
\end{array}\right)\left(\begin{array}{c}
z_{4} \\
w_{2}
\end{array}\right)+\left(\begin{array}{r}
\frac{40}{27} \\
-\frac{10}{27}
\end{array}\right),
$$

which has rest point

$$
\left(z_{4}, w_{2}\right)=-\left(\frac{8}{9}, \frac{38}{135}\right),
$$

characteristic polynomial

$$
p(\lambda)=\left(\lambda^{2}-\frac{5}{3}\right)\left(\lambda^{2}+1\right),
$$

and eigenpairs

$$
\begin{array}{cc}
\lambda_{1}=-1, & R_{1}=\left(\begin{array}{c}
0 \\
1
\end{array}\right) ; \\
\lambda_{2}=\frac{5}{3}, & R_{2}=\left(\begin{array}{c}
\frac{80}{3} \\
-1
\end{array}\right) .
\end{array}
$$

Thus it is a saddle point with linear stable manifold the vertical line $z_{4}=-\frac{8}{9}$, and linear unstable manifold the almost horizontal line

$$
\left(z_{4}, w_{2}\right)=-\left(\frac{8}{9}, \frac{38}{135}\right)+t\left(\frac{80}{3},-1\right) .
$$

\section{Conclusion}

As a first step in authors' program to bring corrections to the Standard Model determined by self-similar waves during the radiation phase $p=\rho c^{2} / 3$ up to present time $p=0$, we have found an elegant asymptotic formulation of equations that close within an ansatz sufficiently general to include these corrections. To our surprise, we discovered a stable rest point $\left(z_{2}, w_{0}\right)=$ $(-4 / 3,1 / 3)$ in the equations for the leading order corrections, the precise order that would introduce a quadratic correction to redshift vs luminosity 
in the Standard Model. We reiterate, the stability of the rest point is not to general perturbations of the equations. It is a more subtle leading order stability to perturbations consistent with self-similar waves from an earlier epoch when mechanisms are in place to create such waves. Our investigations as of the date of this publication indicate that (in the language of [18]), the domain of attraction of this stable rest point appears to contain corrections to the Standard Model coming from self-similar waves with acceleration parameter $a<1$ at the end of the radiation era. These corrections arise from an under-density, a case relevant to the observed anomalous acceleration, but different from the case $a>1$ anticipated to be relevant in [18], c.f. $[6,7,17,18]$. Altogether, this work supplies motivation and clarification for the investigations in authors' forthcoming paper.

\section{References}

[1] A. G. Riess et al. (Supernova Search Team), Observational evidence from supernovae for an accelerating universe and a cosmological constant, Astronomical J. 1163 (1998) 1009-38.

[2] S. Perlmutter et al. (The Supernova Cosmology Project), Measurements of Omega and Lambda from 42 high redshift supernovae, Astrophysical Journal 5172 (1999) 565-86.

[3] M. E. Cahill and A. H. Taub, Spherically Symmetric Similarity Solutions of the Einstein Field Equations for a Perfect Fluid, Commun. Math. Phys. 21 (1971) 1-40. MR0281466

[4] B. J. Carr and A. A. Coley, Self-Similarity in General Relativity, Class. Quantum Grav. 16 (1999) 31-71. MR1701065

[5] C. Copi, D. Huterer, D. J. Schwarz, G. D. Starkman, On the large-angle anomalies of the microwave sky, Mon. Not. R. Astron. Soc. (2005) 1-26.

[6] T. Clifton, P. G. Ferreira, K. Land, Living in a void: testing the Copernican principle with distant supernovae, Phys. Rev. Lett. 101 (2008) 131302 (arXiv:0807.1443v2 [astro-ph] 29 Sep 2008).

[7] T. Clifton and P. G. Ferreira, Does dark energy really exist? Sci. Am. 2009 April (2009) 48-55.

[8] J. Glimm, Solutions in the large for nonlinear hyperbolic systems of equations, Comm. Pure Appl. Math. 18 (1965) 697-715. MR0194770

[9] J. Glimm, P. D. Lax, Decay of solutions of systems of nonlinear hyperbolic conservation laws, Memoirs Amer. Math Soc. 101 (1970). MR0265767 
[10] J. Groah and B. Temple, Shock-wave solutions of the Einstein equations: Existence and consistency by a locally inertial Glimm Scheme, Memoirs of the AMS, Vol. 172, No. 813, November 2004. MR2097533

[11] Ø. Grøn and S. Hervik, Einstein's General Theory of Relativity with Modern Applications in Cosmology, Springer Verlag, 2007. MR2351140

[12] P. D. Lax, Hyperbolic systems of conservation laws, II, Comm. Pure Appl. Math. 10 (1957) 537-566. MR0093653

[13] J. Plebanski and A. Krasinski, An Introduction to General Relativity and Cosmology, Cambridge University Press, (2006). MR2263604

[14] J. Smoller and B. Temple, Global solutions of the relativistic Euler equations, Comm. Math. Phys. 157 (1993) 67-99. MR1234105

[15] J. Smoller and B. Temple, Cosmology, black holes, and shock waves beyond the Hubble length, Meth. Appl. Anal. 11 (2004) 77-132. MR2128353

[16] J. Smoller and B. Temple, Shock-wave cosmology inside a black hole, Proc. Nat. Acad. Sci., September 30, 2003. Vol. 100, no. 20, (1121611218). MR2007847

[17] B. Temple and J. Smoller, Expanding wave solutions of the Einstein equations that induce an anomalous acceleration into the Standard Model of Cosmology, Proc. Nat. Acad. Sci., August 25, 2009, Vol. 106, no. 34, (14213-14218). MR2539730

[18] B. Temple and J. Smoller, General Relativistic Self-Similar Waves that Induce and Anomalous Acceleration into the Standard Model of Cosmology, Memoirs of the AMS, November 3, 2011; S 0065-9266(2011)006416. MR2963331

[19] S. Weinberg, Gravitation and Cosmology: Principles and Applications of the General Theory of Relativity, John Wiley \& Sons, New York, 1972 .

Joel SMOLLer

Department of Mathematics

UNIVERSITY OF MICHIGAN

ANN ARBOR, MI 48104

USA

E-mail address: smoller@umich.edu 
Blake Temple

Department of Mathematics

UNIVERSiTy OF CALIFORNIA

DAVIS, CA 95616

USA

E-mail address: temple@math.ucdavis.edu

ZeKe VogLer

Department of Mathematics

UNIVERSITY OF CALIFORNIA

DAVIS, CA 95616

USA

E-mail address: zekius@math.ucdavis.edu

Received OCtober 1, 2013 